\title{
Towards a Semantic Infrastructure for User Generated Mobile Services
}

\author{
Marcin Davies \\ Telecommunications Research Center Vienna (ftw.), Austria \\ davies@ftw.at
}

\begin{abstract}
This paper presents a research approach towards a semantic infrastructure for user generated mobile services. Building on the concept of semantic microservices, the aim of this work is to sufficiently lower the barrier for end-users in order to enable easy ad-hoc creation, customisation, and discovery of mobile services.
\end{abstract}

Keywords: Semantic web services, end-user development, user-centric service creation, microservices, mobile services, web 2.0.

\section{Introduction and Problem Description}

The current trend of active user participation and service creation on the Internet (Web 2.0, mashups, widgets, etc.) will certainly continue within mobile environments. The enabler technology is mostly available as mobile terminals have an enormous capacity in terms of processing power, interaction, and interfacing. Furthermore, high-speed data coverage as well as flat-fee data plans are increasing to enable an 'always-on' experience in the mobile world.

There is massive potential when mobile devices not only present data but provide valuable information and services to other users. Instant mobile services like traffic jam information, recommended places, or friend locations can be of benefit to a large audience and will pave the way to a new mobile internet empowering end-users to easily find and provide relevant, context-aware information.

Although early approaches towards end-user service creation on the web exist (e.g. Yahoo! Pipes [1]), they cannot be directly transferred to mobile environments (user interface issues, different architecture, etc.). Furthermore, they are too complex for a widespread use (i.e. requiring programming skills) and to facilitate spontaneous service creation on the go.

Driven by the main research question whether and how much semantic technologies can lower the barrier for end-users in order to enable easy ad-hoc creation, customisation, and discovery of mobile services, this thesis introduces the concept of microservices 1 - small, sharply focused, and semantically annotated services that can be flexibly created, adapted, and discovered by end-users. Leveraging this concept, this work will elaborate a semantic infrastructure as a step towards a broad adoption of mobile microservices.

${ }^{1}$ The term also refers to the fact that these services are running on small devices. 


\section{Related Work and Areas of Contribution}

This thesis project will be based on and contribute to the state of the art in three main areas, namely (1) Service Description and Modelling, (2) Knowledge Warehousing and Service Discovery, and (3) End-User Service Creation Paradigms.

\subsection{Service Description and Modeling}

Semantic Web Services (SWS) commonly operate on service descriptions encoded in OWL [2] or RDF [3] and service models such as OWL-S [4] or WSMO 5. In addition, there exist more lightweight approaches such as SAWSDL 6] and SA-REST [7] that put a semantic layer on traditional, non-semantic service description methods such as WSDL [8]. However, none of these techniques are tailored to the needs of mobile services, which are fundamentally different in terms of e.g. life span, context dependency, or service availability. Moreover, existing toolkits for describing SWS (e.g. 9]) are directed towards professionals and are not feasible for end-users while on the go. Therefore, the thesis will explore user-friendly ways of mobile service description and annotation.

Microservice descriptions are interpreted by an execution environment (similar to a virtual machine) on a mobile device and can be shared and customised by other users to create new services. Since existing approaches such as WS-BPEL [10] and model-driven architectures and languages like Executable UML 11] are too much focused on big software systems, this work will present a lightweight microservice model building on the REST [12] architecture that allows for easy integration with existing Web standards and protocols.

\subsection{Knowledge Warehousing and Service Discovery}

A central part of this thesis will consider management and discovery of the previously discussed semantic microservice descriptions. Existing and widespread software like Jena 13. or Sesame 14 allows for arbitrary ontology management, but needs to be enriched by an additional layer supporting community-driven ontologies [15] and ontology matching [16] for microservices and their provided content, revision of knowledge (due to a possibly limited life span of microservices) and content- and context-aware recommendation mechanisms (to deliver meaningful results in mobile scenarios).

\subsection{End-User Service Creation Paradigms and Methods}

Current examples for end-user created software on the Web such as Microsoft Popfly [17] allow users to create new web applications by visual editing of functional blocks. However, these examples can only be regarded as a first step, both in terms of ease of use (still requiring significant knowledge) and functionality (mostly restricted to aggregation of information not provision of it). With respect to the mobile domain, current service creation approaches (e.g. [18[19]) are very limited (again in terms of functionality) or not tailored to mobile devices and usage scenarios (with regard to user interfaces, capabilities, and context information). 
The research area of End-User Development 20] considers different approaches for end-users to create software (e.g. Programming by Example). However, these directions will need to be explored in the context of software services, open communities, and mobile usage patterns. In addition, current end-user development methods are still at an early stage and can benefit from semantic technologies (e.g. by using annotations or rules to describe the behavior of functional blocks).

\section{Methods and Approach}

The overall methodology for the thesis is shown in Fig. 1 and explained in detail below.

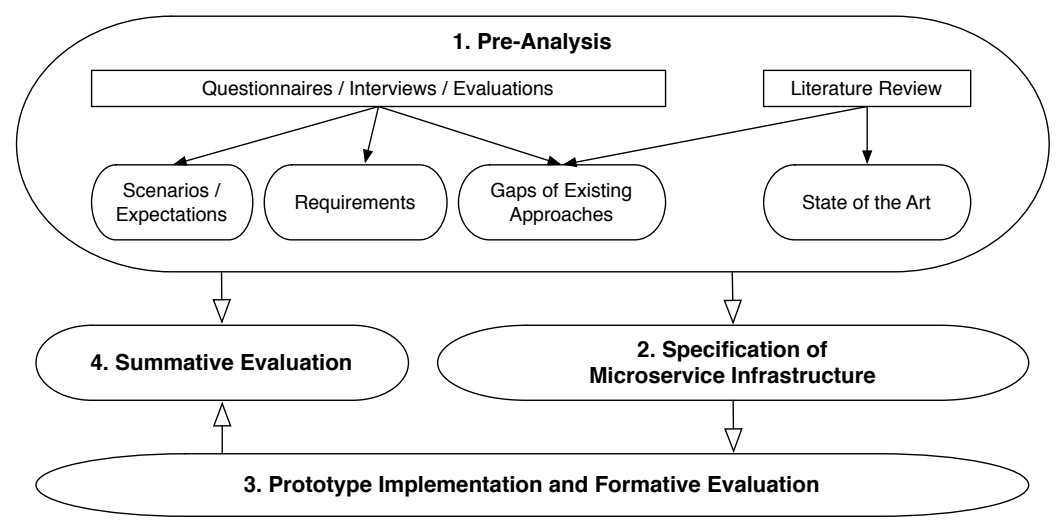

Fig. 1. Overall Methodology

1. Pre-Analysis

In this first phase of the thesis, initial requirements, possible usage scenarios, and user expectations for microservices will be collected by interviews and web questionnaires. In addition, existing frameworks and tools (e.g. LabView 21, Yahoo! Pipes [1, Microsoft Popfly 17]) and small mockups for alternative approaches (e.g. question-based service creation) will be evaluated with regard to specific criteria, e.g. time needed, perceived complexity, and quality of experience (QoX). This pre-analysis should also help to explore gaps in existing solutions that semantic microservices might be able to fill.

2. Specification of Microservice Infrastructure

Based on these results and, of course, review of existing work and the state of the art, a suitable semantic microservice infrastructure will be specified and described. This also includes semantic descriptions of the basic components needed to build microservices.

3. Prototype Implementation and Formative Evaluation

The next step of the thesis will be a prototype implementation of the semantic microservice infrastructure. This framework will consist of serverside software to search and discover microservices (knowledge warehouse) as well as a mobile client that allows for creation, customisation, and execution 
of microservices. By following an iterative design approach, the semantic components derived in early stages of the development will be evaluated in small-scale user studies 2 in order to gather early user feedback and to refine the prototype implementation.

4. Summative Evaluation

Finally, the validity of the developed infrastructure will be assessed in a final user study with regard to easier service creation, customisation, and discovery and by considering the criteria derived in the pre-analysis phase. In addition, the study will also show the applicability to different usage scenarios, e.g. tourism, gaming, recreation, or social networking.

\section{Preliminary Results}

Besides a first definition of scenarios, requirements, and a general system architecture for microservices (cf. 23]), the most relevant achievements so far are towards a specification for the microservice description language. As outlined in Sect. 2.1, microservice descriptions will be used during the whole microservice lifecycle (e.g. creation, modification, execution) and are therefore an essential part of a microservice infrastructure. Currently, the specification foresees the following language parts (more information can be found in [24]):

- Microservice Profile: provides a descriptive and functional definition of the microservice by an extensible set of metadata elements

- Microservice Logic: controls the data flow, capability and content handling, and all other operational aspects of a microservice

- Microservice Rendering: defines the service user interface and provides an abstract and device-independent layer for service presentation

- Microservice Content: describes the content that is manipulated or offered by a microservice

\section{Conclusions and Future Work}

This paper introduced the idea of microservices and described related work and areas of contribution. After outlining the planned approach, this work also presented some preliminary results. According to the methodology shown in Sect. 3 . the next major steps are to complete the pre-analysis phase (already started) in order to receive a sound basis for the infrastructure specification (where work has been started too - cf. Sect. 4). Finally, this infrastructure will be prototypically implemented and evaluated in user studies.

Acknowledgements. This work has been supported by the European Commission, ICT FP7 Collaborative Project m:Ciudad [25]. The Telecommunications Research Center Vienna (ftw.) is supported by the Austrian government and the City of Vienna within the competence center program COMET. Thanks to Anna V. Zhdanova for extensive feedback and supervision of this work.

${ }^{2}$ If needed, 'Wizard-of-Oz' techniques 22 will be applied that simulate behavior that is actually not yet or fully implemented. 


\section{References}

1. Yahoo! Pipes, http://pipes.yahoo.com

2. McGuinness, D.L., Van Harmelen, F.O.: Web Ontology Language Overview, W3C Recommendation (February 10, 2004)

3. Manola, F., Miller, E.: RDF Primer, W3C Recommendation (February 10, 2004)

4. Martin, D., et al.: OWL-S: Semantic Markup for Web Services, W3C Member Submission (November 22, 2004)

5. de Bruijn, J., et al.: Web Service Modeling Ontology (WSMO), W3C Member Submission (June 3, 2005)

6. Farrell, J., Lausen, H.: Semantic Annotations for WSDL and XML Schema, W3C Recommendation (August 28, 2007)

7. Lathem, J., Gomadam, K., Sheth, A.P.: SA-REST and (S)mashups: Adding Semantics to RESTful Services. In: International Conference on Semantic Computing (ICSC), pp. 469-476 (September 2007)

8. Christensen, E., Curbera, F., Meredith, G., Weerawarane, S.: Web Services Description Language (WSDL) 1.1, W3C Note (March 15, 2001)

9. Kerrigan, M., Mocan, A.: The Web Service Modeling Toolkit. In: Bechhofer, S., Hauswirth, M., Hoffmann, J., Koubarakis, M. (eds.) ESWC 2008. LNCS, vol. 5021, pp. 812-816. Springer, Heidelberg (2008)

10. Barreto, C.: WS-BPEL Version 2.0 Primer

11. Mellor, S.J., Balcer, M.J.: Executable UML: A Foundation for Model-driven Architecture. Addison-Wesley, Reading (2002)

12. Fielding, R.T.: Architectural Styles and the Design of Network-based Software Architectures. PhD thesis, University of California, Irvine (2000)

13. Jena: A Semantic Web Framework for Java, http://jena.sourceforge.net

14. SourceForge.net: Sesame, http://sourceforge.net/projects/sesame

15. Zhdanova, A.V.: Community-Driven Ontology Construction in Social Networking Portals. Web Intelligence and Agent Systems 6(1), 93-121 (2008)

16. Zhdanova, A.V., Shvaiko, P.: Community-Driven Ontology Matching. In: Sure, Y., Domingue, J. (eds.) ESWC 2006. LNCS, vol. 4011, pp. 34-49. Springer, Heidelberg (2006)

17. Microsoft Popfly, http://www.popfly.com

18. WidSets: Mobilize Your Web, http://www.widsets.com

19. Shin, Y., Yu, C., Chung, S., Kim, S.: End-User Driven Service Creation for Converged Service of Telecom and Internet. In: Fourth Advanced International Conference on Telecommunications (AICT), pp. 71-76 (June 2008)

20. Lieberman, H., Paternò, F., Wulf, V.: End-User Development. Springer, Heidelberg (2006)

21. National Instruments: LabVIEW, http://www.ni.com/labview

22. Dow, S., MacIntyre, B., Lee, J., Oezbek, C., Bolter, J.: Wizard of Oz Support throughout an Iterative Design Process. IEEE Pervasive Computing (January 2005)

23. Davies, M., et al.: m:Ciudad: An Infrastructure for Creation and Sharing of End User Generated Microservices. In: Poster and Demonstrations Proceedings of the 1st Future Internet Symposium (FIS), Vienna, Austria, pp. 24-26 (September 2008)

24. Carrez, F., et al.: D2.3: m:Ciudad U+ Service Description. Project Deliverable, m:Ciudad ICT FP7 Collaborative Project (January 2009)

25. Project m:Ciudad FP7, http://www.mciudad-fp7.org 